\title{
The nature of geriatrics
}

\section{Interview with Dr Michael Borrie}

\author{
Ramona Neferu (Meds 2018), Han Yan (Meds 2017) \\ Faculty Reviewer: Dr Michael Borrie, BSc, MB ChB, FRCPC (Division of Geriatric Medicine)
}

Dr Michael Borrie is the Program Director of the South Western Ontario Regional Geriatric Program and past Chair of the Division of Geriatric Medicine. Trained in New Zealand and Canada, Dr Borrie shared with us his experiences from 30 years of practice in geriatric medicine and his current roles as a clinician, researcher, administrator, and teacher.

\section{UWOMJ: Tell us a little about yourself.}

Michael Borrie: I did a degree in biochemistry before entering the 5-year medical school training in New Zealand. After medical school, we do 2 years as a "house surgeon", after which we could practice family medicine. I wished to pursue internal medicine and trained in Edmonton, Alberta and then London, Ontario to specialize in geriatric medicine. I met my spouse, Nancy, in my 4 th year in London. We moved to Dunedin, New Zealand where I did 2 years as a research fellow and where we had the first of our three daughters. We returned to London in 1985 . It was very busy doing mainly clinical service on a 40-bed geriatric rehab unit and I was also one of the attending physicians on general internal medicine, doing 1-in-3 call.

\section{What is special about geriatrics?}

I enjoyed internal medicine. Yet, during the subspecialty rotations, I was looking at the patient through the lens of a single organ system. After stabilizing a heart failure for an elderly patient, we were then asked about their arthritis, which we never had the opportunity to address. This was not my idea of patient-centred medicine and not fully satisfying. This confirmed that I wanted to manage older people, multi-system disease and the related complexities, and the field of geriatric medicine was clearly for me.

Geriatric medicine is special because you can analyze an event such as a fall and split out the multiple factors. Often, 6 or more different factors combine to cause a person to eventually fall. That is the nature of geriatrics. You have a single syndrome: falls, confusion, incontinence or polypharmacy. You can dissect it out and ask "what are all the reversible and irreversible factors" and then focus on the reversible elements.

\section{What do you like most about being a geriatrician?}

It's a very satisfying career. Older patients are very appreciative of what you do and they're very respectful. They don't always follow advice but that's no different than other patients. I can't change the world but I can do multiple small things for their many complex diseases to make a difference to their quality of life and, ultimately, this is what medicine comes down to-improving the quality of someone's life.
Geriatric medicine deals with multiple chronic diseases and their complex interaction. I enjoy the intellectual stimulation of understanding a person's history, as well as the clinical examination and investigations. I have the luxury of time for a full history, examination, and comprehensive counselling that may make a difference.

Research has always interested me. There are many research opportunities in geriatric medicine from the basic science of individual diseases, to clinical trials of novel compounds, to knowledge translation that changes patterns of clinical practice and mindful clinical care.

Our division is one of the largest and well-integrated divisions across the country. We can support some of us to lead as clinical educators or clinician-scientists and everything in between. Each of the geriatricians pursues his or her individual strengths and works together with allied health professional teams to exemplify best practices, care, and education for older adults in our region.

\section{What are your current responsibilities?}

I do approximately equal amounts of research, administrative work, and clinical work, as well as clinical teaching in the outpatient clinic or on the geriatric rehabilitation unit.

In 1985, I began my career as a geriatrician and by 1995, became the chair of the division. I was one of 4 geriatricians and over the next 15 years took on the leadership and administrative role of the division. We recruited geriatricians and built the division to 11 members. As an academic geriatrician, you can develop as a clinician, researcher or clinician-teacher, although it should be noted that it is difficult to do both clinic work and research.

Through my training, interests and opportunities in Canada, I have evolved as a clinician-researcher. My research focus was initially on Alzheimer disease and also on urinary incontinence. In the early 1990s, we began new trials in Alzheimer disease and offered participation in these clinical trials to patients seen in the Aging Brain and Memory Clinic. Over the last 10 years, the focus has been on treatments for the amyloid hypothesis and disease modification. The trials are more complex lasting several years and require positron emission tomography imaging for amyloid.

Can you outline the 2+1 Care of the Elderly option vs 5-year training in Geriatric Medicine?

Family physicians with an extra year in Care of the Elderly can be very effective physicians in geriatric care. The longer they practice in geriatric care, the more the distinction between their clinical practice and that of a geriatrician narrows. 
Care of the Elderly-trained physicians who pursue an academic career are more likely to have an academic role as educators rather than researchers, unless they have additional research training. In community practice they may be the physician lead providing consultation or direct management for the elderly patients within a family health team. They may pursue the Care of the Elderly training to develop additional skills for the management of elderly patients within their practice.

As you complete your medical degree, if you are unsure if you want a career in family medicine with geriatrics as a focus or a career in geriatric medicine, entering an internal medicine program is always a good idea. It is easier to switch into family medicine from internal medicine than the reverse, and you will be a better physician for having done additional training in internal medicine.

In Ontario, of the 130 geriatricians who have completed the subspecialty 5 -year training in geriatric medicine, half are in academic centres and half are in community settings.

\section{What are the opportunities and challenges in the future for geri- atrics?}

My bias is towards the opportunity to prevent, manage and treat cognitive impairment. Cognitive impairment is the one condition that threatens a person's independence. Right now the direct and indirect costs due to cognitive impairment/dementia are about $\$ 10$ billion a year. By 2038 , the costs are estimated to be $\$ 150$ billion per year. The biggest driver of direct costs is people with cognitive impairment staying in acute care beds and eventually having to live in long term care institutions. We could do so much more to prevent cognitive decline and its progression.

Historically, acute care hospitals were built to treat acute illnesses. We can do much better at preventing delirium following surgery or medical interventions. There is an irreversible component of delirium and patients will deteriorate during an acute care admission. This will result in longer hospital stay and drive up our health care costs.

The challenge to future physicians will be developing smarter models of care. Technology will affect health care and you will have very tech-savvy elderly patients in your practice. For example, as a consultant, communication will include e-consults and there will need to be different funding models to support this.

\section{What are your hobbies or personal interests?}

My wife and I love to regularly do social and Latin dancing. We've been doing this since 2005. It's good for our brain and our relationship. I've always enjoyed swimming-in high school as a competitive swimmer-and now recreationally whenever I have the opportunity.

\section{Any advice for medical students interested in geriatrics?}

Take advantage of any opportunities for clinical rotations and exposure to geriatric medicine during your training. Get research experience in geriatrics, such as the summer research training program.

Do you have any thoughts about the recent ruling in favour of legalizing physician-assisted suicide (PAS)?

In 30 years of practice, I cannot think of a patient encounter where I've said that the only option at this point is assisted suicide. That may mean that people who would benefit from PAS have never come to a geriatrician. There may well be a few people for whom assisted suicide might be the only option. However, I think one has to be cautious about assisted suicide and the potential for a slippery slope. If it is thought through very carefully and there are appropriate policies and safeguards in place, there may be some people who could benefit. Most people with severe pain are managed appropriately and assisted suicide does not have to be considered. As a society, we need to protect people who are vulnerable, particularly older individuals with disabilities or cognitive impairment, so that they don't feel they have to "move on" and do the honourable thing. Fortunately for me, in geriatric medicine, I have never felt the need to consider this option. I work with teams and when appropriate we aim to provide the best end-of-life care. 